\title{
Characterization of a novel type III CRISPR-Cas effector provides new insights into the allosteric activation and suppression of the Cas10 DNase
}

\author{
Jinzhong Lin (1) ${ }^{1}$, Mingxia Feng ${ }^{1,2}$, Heping Zhang ${ }^{3}$ and Qunxin She ${ }^{4}$
}

\begin{abstract}
Antiviral defense by type III CRISPR-Cas systems relies on two distinct activities of their effectors: the RNA-activated DNA cleavage and synthesis of cyclic oligoadenylate. Both activities are featured as indiscriminate nucleic acid cleavage and subjected to the spatiotemporal regulation. To yield further insights into the involved mechanisms, we reconstituted LdCsm, a lactobacilli III-A system in Escherichia coli. Upon activation by target RNA, this immune system mediates robust DNA degradation but lacks the synthesis of cyclic oligoadenylates. Mutagenesis of the Csm3 and Cas 10 conserved residues revealed that Csm3 and multiple structural domains in Cas10 function in the allosteric regulation to yield an active enzyme. Target RNAs carrying various truncations in the $3^{\prime}$ anti-tag were designed and tested for their influence on DNA binding and DNA cleavage of LdCsm. Three distinct states of ternary LdCsm complexes were identified. In particular, binding of target RNAs carrying a single nucleotide in the 3' anti-tag to LdCsm yielded an active LdCsm DNase regardless whether the nucleotide shows a mismatch, as in the cognate target RNA (CTR), or a match, as in the noncognate target RNA (NTR), to the $5^{\prime}$ tag of crRNA. In addition, further increasing the number of 3' anti-tag in CTR facilitated the substrate binding and enhanced the substrate degradation whereas doing the same as in NTR gradually decreased the substrate binding and eventually shut off the DNA cleavage by the enzyme. Together, these results provide the mechanistic insights into the allosteric activation and repression of LdCsm enzymes.
\end{abstract}

\section{Introduction}

CRISPR-Cas (clustered regularly interspaced short palindromic repeats (CRISPR)-CRISPR-associated (Cas)) systems constitute the adaptive and heritable immune system in bacteria and archaea, which mediates antiviral defense against invasive genetic elements in a small RNAguided fashion ${ }^{1-8}$. The immune system consists of two parts: CRISPR arrays containing spacers derived from invading nucleic acids, and cas gene cassettes coding for enzymes or structural proteins that function in mediating

Correspondence: Jinzhong Lin (jinzhong.lin@bio.ku.dk) or

Qunxin She (shequnxin@sdu.edu.cn)

${ }^{1}$ Archaea Centre, Department of Biology, University of Copenhagen, Ole Maaløes Vej 5, 2200 Copenhagen N, Denmark

${ }^{2}$ College of Life Science and Technology, Huazhong Agricultural University, Wuhan, China

Full list of author information is available at the end of the article the CRISPR immunity. CRISPR-Cas systems are classified into two broad classes based on the composition of their effector complexes: those of Class 1 carry multi-subunit effectors and those of Class 2 possesses a single effector protein, and these antiviral systems are further divided into six main types (types I-VI) with $>20$ subtypes $^{9-11}$.

Type III CRISPR systems are unique because they exhibit both RNA interference and DNA interference in vivo to protect their microbial hosts against invading nucleic acids $^{4,12-23}$. Three activities are associated with these type III immune systems, including target RNA cleavage ${ }^{18,24-30}$, target RNA-activated indiscriminate single-stranded (ss) DNA cleavage, a secondary DNase activity ${ }^{15,19,20,31-37}$, and synthesis of cyclic oligoadenylate ( $\mathrm{cOA})$, a second messenger that activates RNases of Csm6/Csx1 families, mediating cell dormancy or cell death ${ }^{38-45}$. Activation of the immunity

\section{(c) The Author(s) 2020}

(c) Open Access This article is licensed under a Creative Commons Attribution 4.0 International License, which permits use, sharing, adaptation, distribution and reproduction c. in any medium or format, as long as you give appropriate credit to the original author(s) and the source, provide a link to the Creative Commons license, and indicate if changes were made. The images or other third party material in this article are included in the article's Creative Commons license, unless indicated otherwise in a credit line to the material. If material is not included in the article's Creative Commons license and your intended use is not permitted by statutory regulation or exceeds the permitted use, you will need to obtain permission directly from the copyright holder. To view a copy of this license, visit http://creativecommons.org/licenses/by/4.0/. 
requires mismatches between the $3^{\prime}$ anti-tag of a cognate target RNA (CTR) and the $5^{\prime}$ tag of crRNA, whereas the full match between the $3^{\prime}$ anti-tag of noncognate target RNA (NTR) and the $5^{\prime}$ tag of crRNA completely represses the immune response (see reviews ${ }^{46-49}$ ). Upon activation by the secondary messenger, Csx1/Csm6 RNases exhibit indiscriminate cleavage of viral and cellular RNAs, leading to cell dormancy or cell death to curb virus infection ${ }^{38-43,50,51}$. It is further believed that the type III CRISPR DNase eventually clears up remaining invading nucleic acids ${ }^{52}$, whereas the cOA secondary messenger is to be removed by ring nucleases $^{53}$, allowing cells recover from the type III immune response and restore the growth.

Structure of the Streptococcus thermophilus III-A (StCsm) effector complex has recently been resolved. This includes that of the StCsm binary complex and those of the $\mathrm{StCsm}^{\mathrm{CTR}}$ and StCsm ${ }^{\mathrm{NTR}}$ ternary effector complexes. The two ternary complexes have undergone major conformational change, relative to the binary one, but the active $\mathrm{StCsm}^{\mathrm{CTR}}$ complex and the inactive $\mathrm{StCsm}^{\text {NTR }}$ complex show minimal conformational change except for the fact that the $3^{\prime}$ anti-tag of CTR and that of NTR are placed in different channels ${ }^{54}$. Very similar results were obtained from the structural analysis of the Thermococcus onnurineus Csm effector complex ${ }^{55}$ and the StCsm effector of different composition ${ }^{56}$. In particular, the active site of these Csm DNases exhibits little difference between the NTR-bound effector complex and the corresponding CTR-bound effector complex. As a result, the process of the activation of a binary Csm complex by CTR as well as its inhibition by NTR remains elusive.

To gain an further insight into the molecular mechanisms of the allosteric regulation of type III-A effector complexes, we characterized a Csm present in Lactobacillus delbrueckii subsp. bulgaricus (LdCsm). The effector complex was reconstituted by the expression in, and purified from, Escherichia coli. Investigation of the interactions between purified effector complexes and their target RNAs unravels an active ternary LdCsm complexes with CTR or NTR carrying a single nucleotide at their $3^{\prime}$ anti-tag regions, and these results provide a novel insight for allosteric activation and repression of the LdCsm DNase.

\section{Results}

\section{L. delbrueckii subsp. bulgaricus encodes a novel III-A CRISPR-Cas system defective in COA synthesis}

The $L$. delbrueckii subsp. bulgaricus strain carries a type III-A CRISPR-Cas system (LdCsm) including a CRISPR array of 16 spacers (Fig. 1a), in addition to a type II CRISPR-Cas9 system in another chromosome location (GenBank: CP016393.1). This LdCsm system was chosen for characterization since phylogenetic analyses of a selected set of Cas10 proteins revealed that its Cas10, the L. delbrueckii subsp. bulgaricus Csm1, is distantly related to the Cas10 proteins that have been studied thus far (Supplementary Fig. S1).

We chose to reconstitute the LdCsm effector in E. coli since recombinant protein purification procedure has not been established for this lactobacillus yet. Three different E. coli vectors, i.e., p15AIE, pUCE, and pET30a, were employed to clone all components of the immune system, including the complete set of III-A cas genes, a His-tag version of $\operatorname{csm} 2$, and a synthetic CRISPR array (Supplementary Fig. S2). Expression of these plasmid-borne genes in the same cell yielded all the III-A Cas proteins, a Histagged LdCsm2 protein, and crRNAs (Fig. 1b), which were allowed to assemble into recombinant LdCsm ribonucleoprotein complexes in E. coli. The resulting effector complexes were then purified in two-step purification, the nickel-His tag affinity chromatography and the size exclusion chromatography (SEC). A single protein peak appeared in 10-12 $\mathrm{ml}$ in the SEC purification (Fig. 1c), indicative of copurification of large complexes. Analysis of these SEC samples by sodium dodecyl sulfatepolyacrylamide gel electrophoresis (SDS-PAGE) showed that each SEC sample contained five protein bands corresponding to the predicted sizes of Csm1, Csm2, Csm3, Csm4, and Csm5, respectively (Fig. 1d). The RNA component was extracted from the effector complexes using the Trizol agent. Denaturing PAGE analysis of extracted RNAs by radio-labeling and northern blot analysis of these RNAs by the radio-labeled DNA probe revealed 3 major RNA components of $\sim 76,38$, and 32 nucleotides (nts). The largest species represented the complete unit of S1 crRNA carrying the $5^{\prime}$-repeat handle $+\mathrm{S} 1$ spacer +3 'repeat handle, representing the Cas6-cleaved crRNA product of a single spacer, whereas the two smaller RNA species were matured crRNAs (Fig. 1e and Supplementary Fig. S3). Together, these results indicated that LdCsm ribonucleoprotein complexes were reconstituted in E. coli. Since F5-F7 fractions mainly contained the LdCsm complex of 32 nt crRNA (Fig. 1e), the smallest ribonucleoprotein effector complex, they were pooled together, annotated as LdCsm, and characterized.

To test whether the $E$. coli-expressed effector could be active in RNA cleavage, LdCsm was mixed with four different radio-labeled RNAs individually, i.e., (a) a nonhomologous RNA, S10, (b) the protospacer target RNA (PTR), S1-40, lacking any 3' anti-tag, (c) the CTR, S1-46, exhibiting mismatches between its $6 \mathrm{nt} 3^{\prime}$ anti-tag and the $5^{\prime}$ tag of the corresponding crRNA, and (d) the NTR, S1-48, possessing the fully complementary sequence between its $3^{\prime}$ anti-tag and the $8 \mathrm{nt} 5^{\prime}$ tag of the crRNA (Fig. 2a). These reaction mixtures were incubated at $37^{\circ} \mathrm{C}$ for $10 \mathrm{~min}$ and analyzed by denaturing PAGE. We found that, while the non-homologous RNA was not a substrate of LdCsm (Supplementary Fig. S4a), the effector cleaved all three homologous target RNAs in 6-nt periodicity with similar 


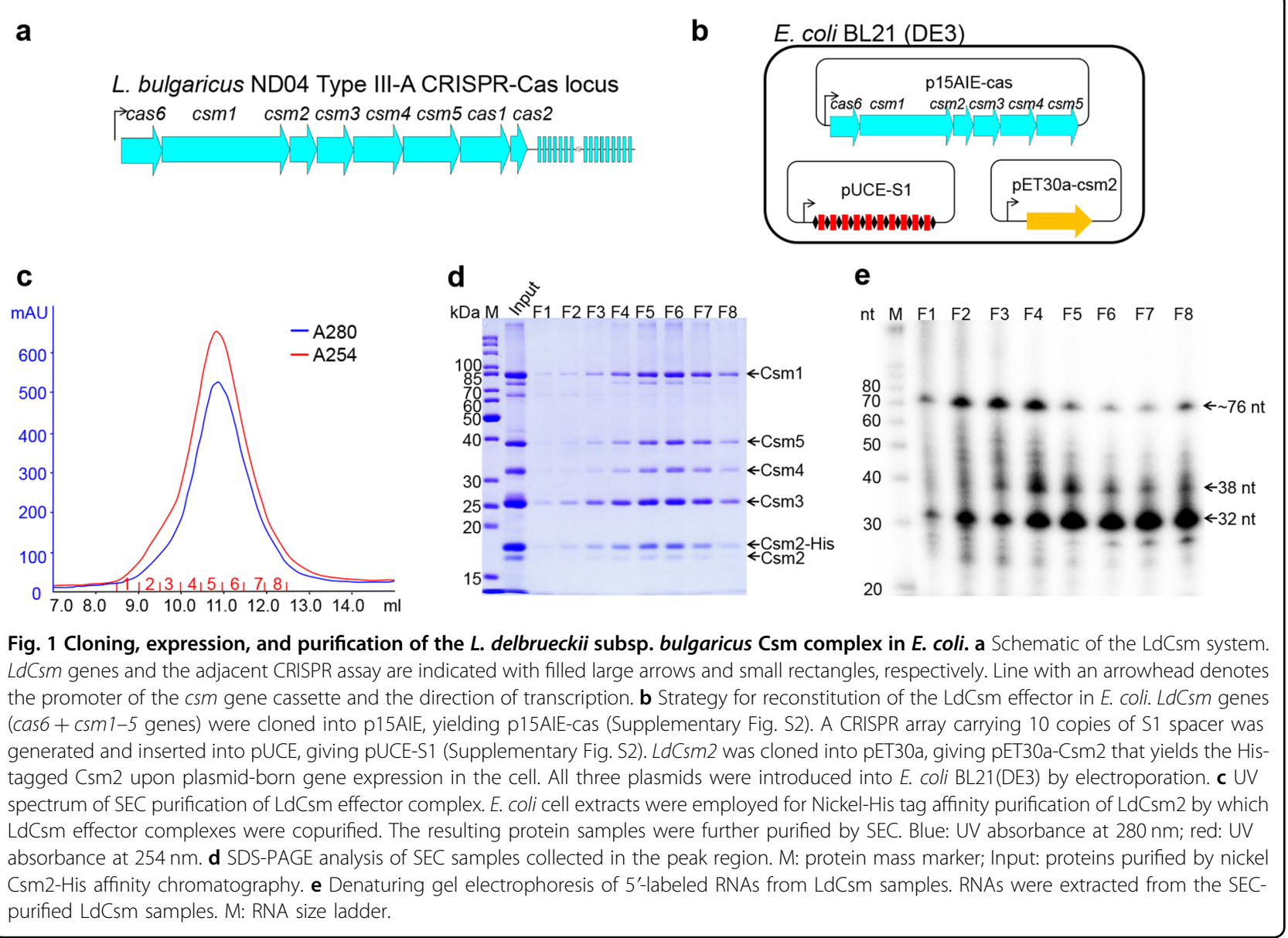

efficiencies. These results indicated that LdCsm possesses the backbone RNA cleavage activity and $3^{\prime}$ anti-tag sequences on target RNAs do not influence the RNA cleavage (Fig. 2b). Then all three target RNAs were tested for their capability of mediating RNA-activated DNA degradation to S10-60, a single-stranded non-homologous DNA substrate (Supplementary Table S1). As shown in Fig. 2c, only CTR activated the LdCsm DNase, indicating that mismatches between the $3^{\prime}$ anti-tag of the target RNA and the corresponding $5^{\prime}$ tag of crRNA are essential for the activation. These results are in good agreement with those reported for other characterized type III CRISPR-Cas systems $^{15,17,19,20,31,34,35}$. We further tested the minimal concentration of CTR is required for the ssDNase activity of LdCsm. To do that, a fluorophore quencher-labeled 16-nt poly-dT ssDNA reporter (FAM-poly-16T-BHQ1) containing a 5'-FAM (5'-carboxyfluorescein) and 3'-BHQ1 (3'Black Hole Quencher-1) was synthesized and employed as ssDNA substrate. Reactions containing $50 \mathrm{nM} \mathrm{LdCsm}$ and a wide range of concentrations of CTR $(0-10 \mathrm{nM})$ were set up using a microplate. the result showed that $0.5-1 \mathrm{nM}$ CTR is efficient to activate the ssDNase activity of LdCsm (Supplementary Fig. S5).
To date, all studied type III effectors are capable of producing cOAs, a secondary messenger that activates CRISPR-associated Rossmann fold domain RNases of the Csm6/Csx1 family for general degradation of cellular RNAs $^{38-42}$. To investigate whether LdCsm could also do that, the effector was mixed with ATP and CTR in the presence of $\mathrm{Mg}^{++}$and incubated for $2 \mathrm{~h}$. Samples were analyzed by denaturing PAGE. As shown in Fig. 2d, whereas Sulfolobus islandicus III-B Cmr- $\alpha$ (a positive reference $^{38}$ ) consumed almost all $100 \mu \mathrm{M}$ ATP for cOA synthesis, LdCsm did not produce any detectable cOA in the same reaction set-up. We repeated the experiments several times and also tested with a number of different metal ions but constantly failed to detect any cOA in the ATP reaction (Supplementary Fig. S4f).

We noticed that LdCsm1 carries a QGDD motif in Palm 2 , the active site for cOA synthesis, which differs from the GGDD consensus (Supplementary Fig. S6a). To test whether the occurrence of glutamine (Q597) residue in the motif could be responsible for cyclase inactivation in LdCsm, a Q597G substitution mutant was constructed to restore the common GGDD motif in LdCsm1, denoted $\mathrm{Csm1} 1^{\mathrm{Q} 597 \mathrm{G}}$. Characterization of the resultant mutated 


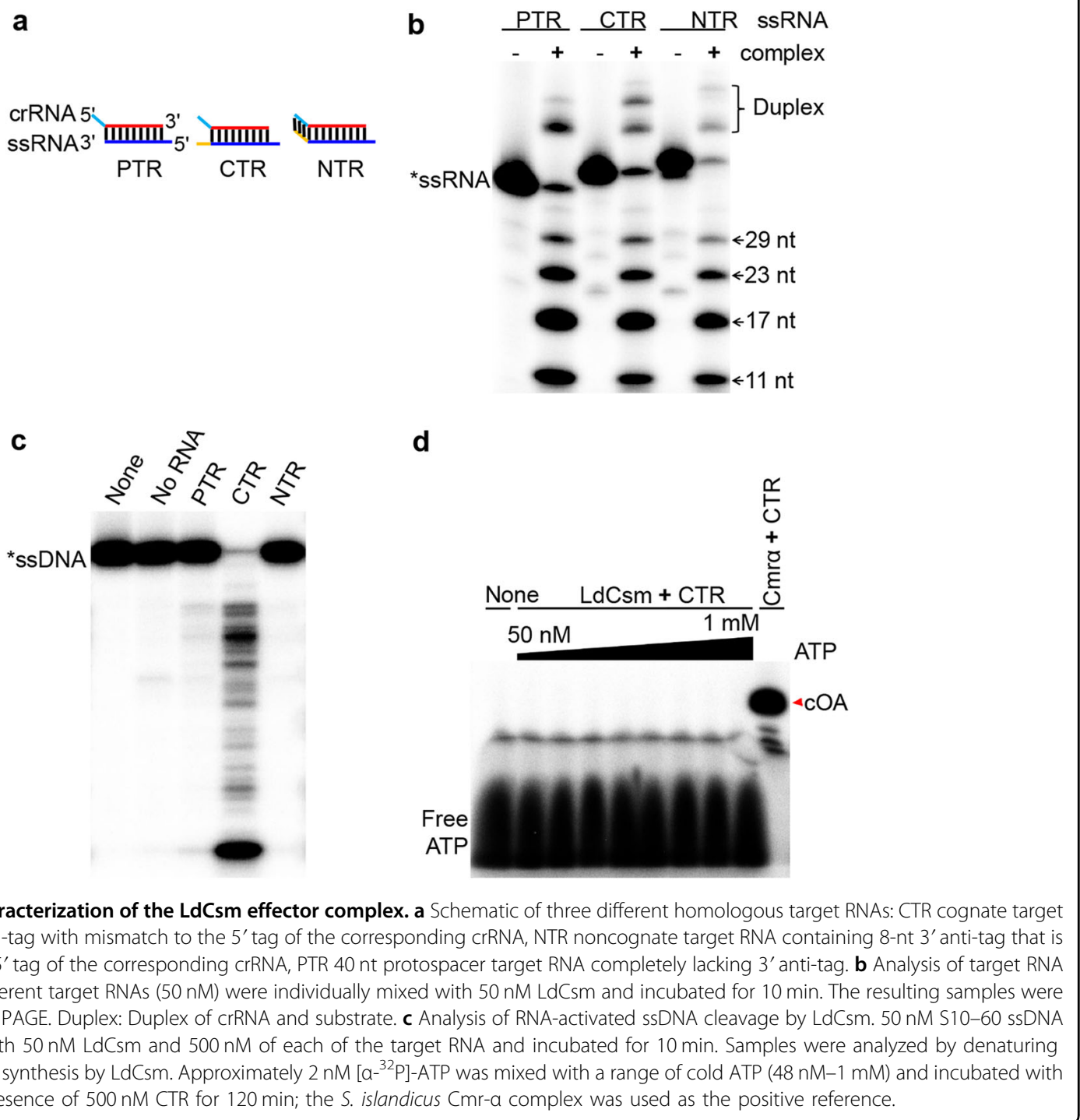

effector revealed that the GGDD-restored LdCsm still appeared to be inactive in cOA synthesis (Supplementary Fig. S4f). To this end, the LdCsm cyclase inactivation must not have resulted from any spontaneous mutation at the Cas10 cyclase active site.

LdCsm system mediates anti-plasmid interference in $E$. coli

To investigate whether the LdCsm system could mediate DNA interference in vivo, three test plasmids were constructed using pBad, an $E$. coli vector exhibiting arabinose-inducible expression. These included pBadeGFP (pBad-G), a reference plasmid, and two protospacer-carrying plasmids: one containing the CTR$\mathrm{S} 1$ protospacer-GFP fusion gene (pBad-CTR), and the other possessing the NTR-S1 protospacer-eGFP (pBadNTR) (Fig. 3a). Meanwhile, E. coli strain for the in vivo assay was generated by introduction of the expression plasmid p15AIE-Cas-S1 into E. coli BL21(DE3) by electroporation. The rationale of the experiment is that plasmid-borne gene expression from p15AIE-Cas-S1 would yield binary LdCsm effector complexes in the host cells, which would mediate anti-plasmid activity if a CTR was to be expressed from a test plasmid. In addition, since the expression of CTR-S1 or NTR-S1 target RNA is controlled by the Bad promoter that confers very stringent arabinose-inducible expression, target RNAs would only be synthesized in L-arabinose media but not in glucose media. Therefore, examination of colonyformation efficiency of each plasmid on both arabinose plates and glucose media would reveal in vivo transcription-dependent anti-plasmid interference of the immune systems to be tested (Fig. 3b).

All test plasmids were introduced into the genetic host by transformation, and transformed $E$. coli cells were 


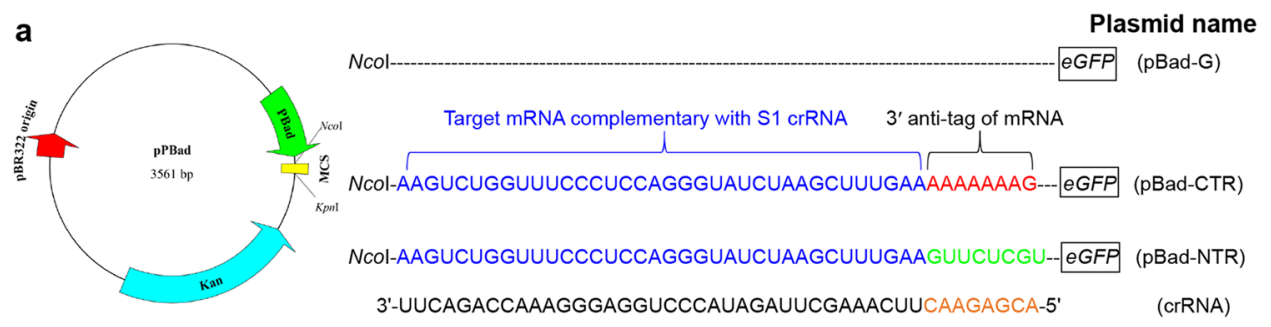

b

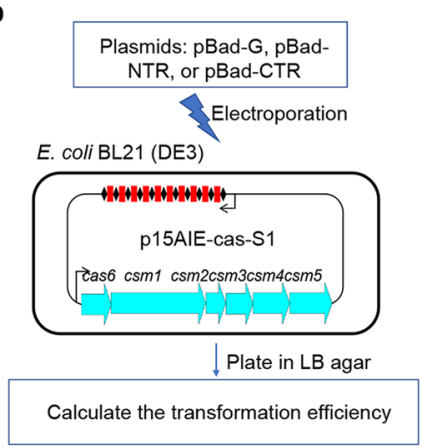

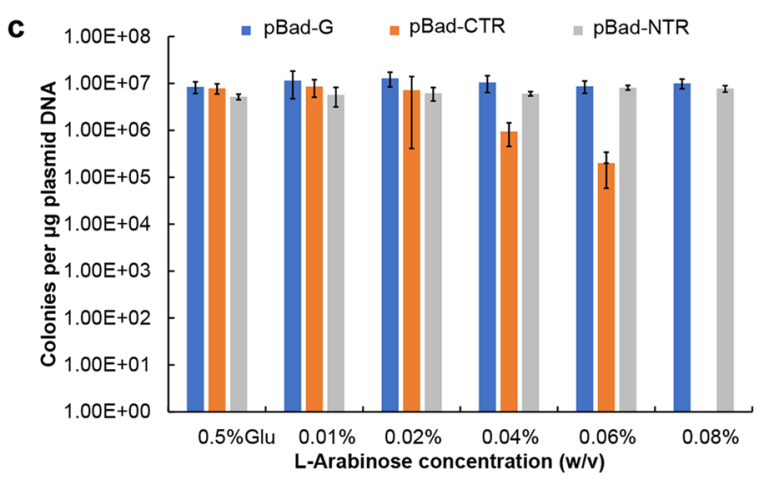

Fig. 3 Anti-plasmid activity of the LdCsm system. a Schematic of the test plasmids pBad-G, pBad-CTR, and pBad-NTR. Gene expression from these plasmids is under the control of a L-arabinose-inducible Bad promoter. Therefore, mRNAs carrying CTR or NTR are expressed from the corresponding plasmids (pBad-CTR or pBad-NTR) in the presence of L-arabinose, but their expression is completely repressed in glucose media. $\mathbf{b}$ Schematic of the interference plasmid assay for determination of the LdCsm anti-plasmid activity. c Transformation efficiency data of the three different plasmids obtained from different growth media.

plated on 6 different medium plates containing $0.5 \%$ glucose or arabinose of different contents (0.01-0.08\%). Following transformation data were obtained: (a) pBad-G and pBad-NTR produced very similar numbers of transformants on all 6 growth media, (b) pBad-CTR gave very similar transformation efficiency data on plates containing glucose or very low concentrations of L-arabinose $(0.01 \%$ or $0.02 \%)$, (c) increasing the L-arabinose content in the medium to $0.04 \%$ and $0.06 \%$ yielded ca. 10 - and 100 -fold decrease in transformation efficiency, and (d) the presence of $0.08 \%$ inducer completely abolished colony formation by pBad-CTR-containing cells (Fig. 3c). These results indicated, while NTR can effectively turn off the LdCsm immunity, CTR is capable of conferring plasmid clearance to the LdCsm system albeit it only possesses the RNAactivated ssDNA cleavage activity. This is in contrast to the current knowledge of antiviral mechanisms by type III CRISPR-Cas systems in which the cOA signaling pathway plays a main role in the immunity ${ }^{13,51,52,57,58}$, suggesting that LdCsm represents the novel type III CRISPR-Cas system, which exhibits the robust RNA-activated DNase activity to resist the invasive plasmid.

\section{Multiple Cas10 domains contribute to the LdCsm DNase activity}

The largest subunit Cas10 in type III-A effector complexes contains four conserved domains, including a HD domain, two Palm domains (Palm1 and Palm2), a Linker, and a D4 structural region (Fig. 4a). Biochemical and structural analyses of different type III CRISPR-Cas systems have revealed that the HD domain is responsible for ssDNA cleavage ${ }^{15,19,20,31,34,35}$, and Palm1 and Palm2 domains function in cOA synthesis ${ }^{38-41,43}$, whereas the Linker domain plays a regulatory role in both target RNA-activated DNA cleavage and cOA synthesis ${ }^{54}$. However, it remains unclear whether a concerted action of different Cas10 domains would be required to yield Csm DNase with optimal activity. The robustness of the LdCsm DNase rendered it a good system for this characterization.

Nine conserved amino acids present in the HD, Linker, and Palm2 domains of LdCsm1 were chosen for substitution mutagenesis, giving six single domain mutants. These included Csm $1^{\mathrm{dHD}}$, a HD domain mutant carrying H15A and D16A double substitution, 3 Linker domain mutants, i.e., $\mathrm{Csm} 1^{\mathrm{LinE}}$ (E415A substitution), $\mathrm{Csm} 1^{\mathrm{LinEC}}$ (E415A and C416A substitutions), and $\mathrm{Csm}^{\mathrm{LinCC}}$ (C416A and C419A mutations), and 2 Palm2 domain mutants, Csm1 ${ }^{\mathrm{P} 2 \mathrm{DxD}}$ (D541A and D543A substitutions) and $\mathrm{Csm} 1^{\mathrm{P} 2 \mathrm{DD}}$ (D599A and D600A mutations). Then some mutated motifs were combined, yielding double domain mutations, including two $\mathrm{HD} /$ Linker mutants $\left(\mathrm{Csm} 1^{\text {dHD_LinEC }}\right.$ and Csm1 $\left.1^{\mathrm{dHD} \_ \text {LinCC }}\right)$, two HD/Palm2 mutants (Csm1 $1^{\text {dHD_P2DxD }}$ and Csm1 $1^{\text {dHD_P2DD }}$ and 
a

c

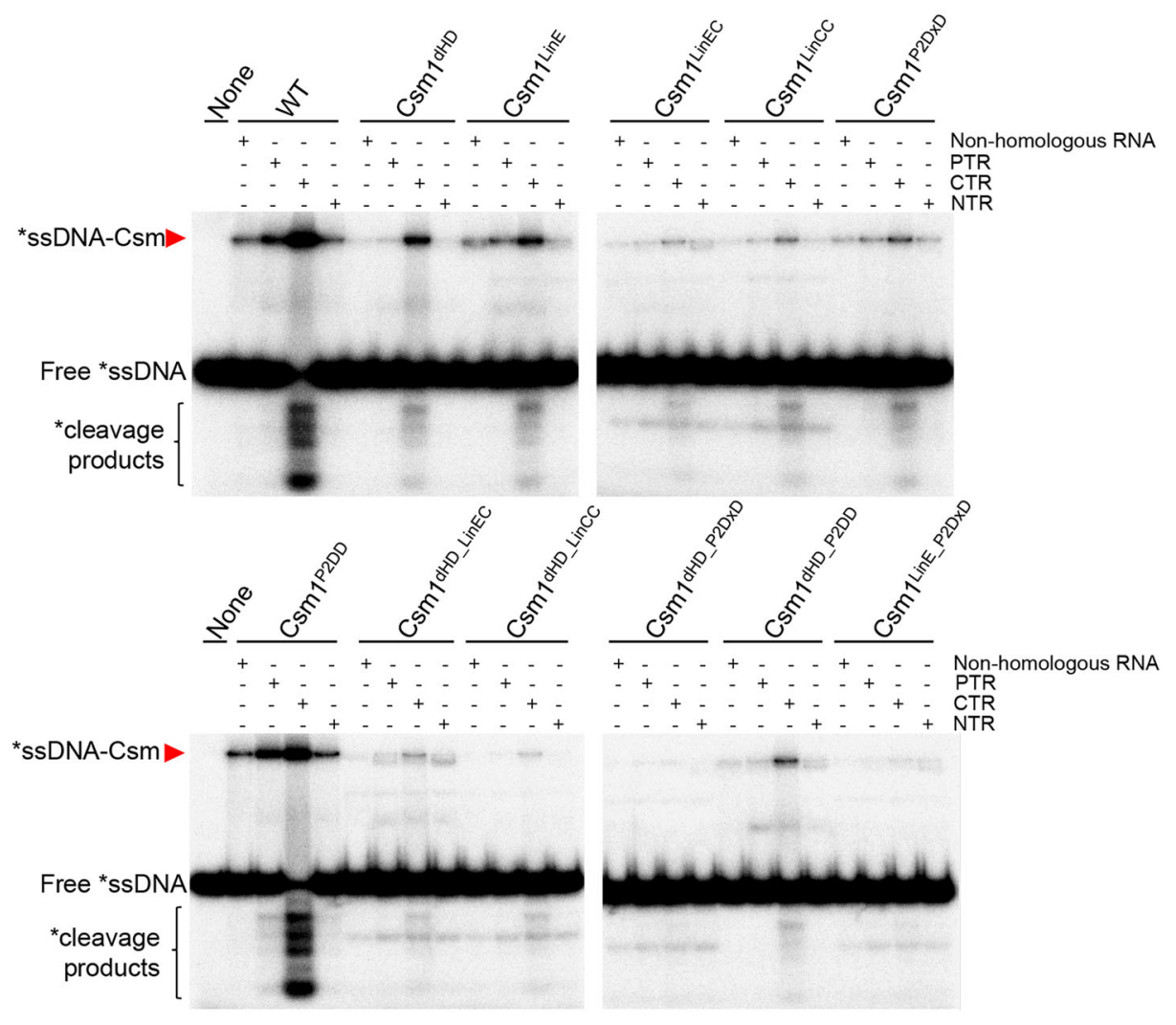

d

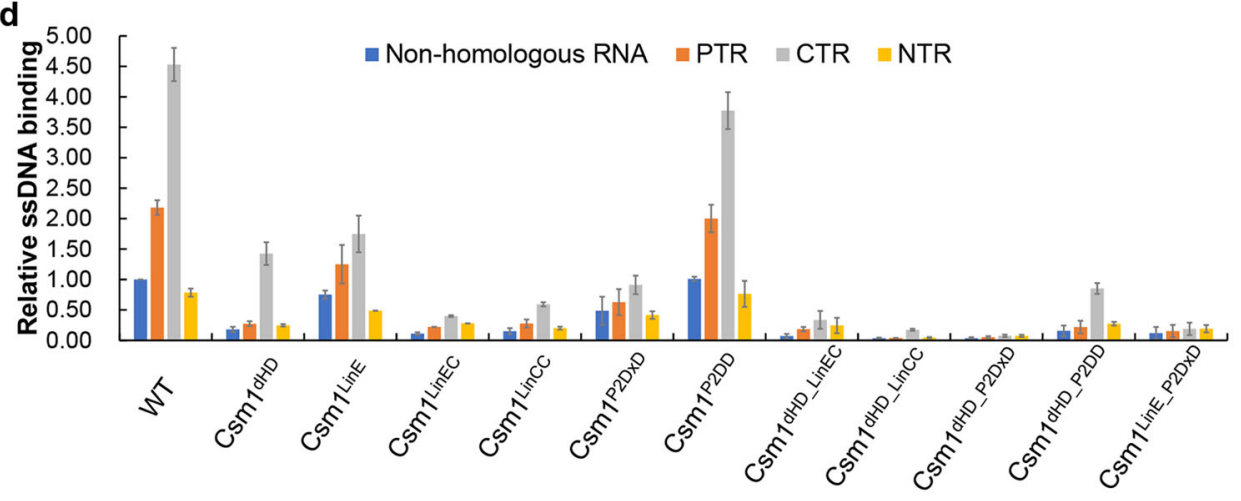

Fig. 4 (See legend on next page.) 
(see figure on previous page)

Fig. 4 Effect of LdCsm1 mutations on ssDNA binding and cleavage by the LdCsm effector complex. a Domain architecture of the LdCsm1 protein. HD represents the HD-type nuclease domain; Palm 1 and Palm 2 denote the two cyclase domains; Linker is a domain that adjoins the Palm 1 and Palm2 domains, consisting of four cysteine residues; D4 is located in the C-terminus rich in a-helices. Amino acid residues selected for alanine substitution mutagenesis are indicated with their names and positions. $\mathbf{b}$ RNA-activated ssDNA cleavage by effectors carrying one of the constructed LdCsm1 mutants. Fifty nM S10-60 ssDNA substrates were mixed with 50 nM mutated LdCsm carrying each of LdCsm 1 mutant proteins and $500 \mathrm{nM}$ CTR and incubated for $10 \mathrm{~min}$. Samples were analyzed by denaturing PAGE. c ssDNA binding by effectors carrying each of the constructed LdCsm1 mutants. Five nM labeled S10-60 ssDNA were incubated for 3 min with $100 \mathrm{nM}$ of LdCsm effectors in the presence of $400 \mathrm{nM}$ of non-homologous RNA (S10 RNA) or $500 \mathrm{nM}$ of one of the target RNAs, PTR or CTR or NTR. Samples were analyzed by non-denaturing PAGE. Red arrowheads indicate the Csm-ssDNA complex. $\mathbf{d}$ Relative ssDNA binding between the wild-type LdCsm effector and its LdCsm1 mutated derivatives. The relative ssDNAbinding activities were estimated by image quantification of the non-denaturing PAGE in $\mathbf{c}$ by the accessory analysis tool in Typhoon FLA 7000, the ssDNA activity of LdCsm in non-homologous RNA was used as the standard and set up as 1. Results shown are average of three independent assays; bars represent the mean standard deviation $( \pm S D)$.

Csm1 $1^{\text {LinE_P2DxD }}$, a Linker, and a Palm2 mutant (Supplementary Table S2).

Each mutated $\operatorname{csm} 1$ gene was used to replace the wildtype (WT) $c s m 1$ on p15AIE-cas, and the resulting plasmids were introduced into $E$. coli to express LdCsm effectors carrying each of the mutated LdCsm1 protein. These mutated LdCsm effector complexes were obtained as described for the WT LdCsm effector. The protein components of these effector complexes were checked by SDSPAGE, and this showed that they all contained five different Csm subunits as for the WT LdCsm complex (Supplementary Fig. S6b), indicating that none of these Csm1 mutations affected the effector assembly. These effector complexes were then tested for target RNA cleavage using S1-46, the CTR, as the substrate, and they all exhibited the backbone cleavage (Supplementary Fig. S6c).

Next, RNA-activated DNase activity was examined for each mutated complex in reactions containing $50 \mathrm{nM}$ effector, $50 \mathrm{nM}$ ssDNA, and $500 \mathrm{nM}$ CTR. For single domain mutants, we found that all mutations (except for $\mathrm{Csm}^{\mathrm{P} 2 \mathrm{DD}}$ ) strongly impaired the ssDNase activity, including $\mathrm{Csm} 1^{\mathrm{dHD}}, \mathrm{Csm} 1^{\mathrm{LinE}}, \mathrm{Csm} 1^{\mathrm{LinEC}} \mathrm{Csm} 1^{\mathrm{LinCC}}$, and $\mathrm{Csm}^{\mathrm{P} 2 \mathrm{DxD}}$ (Fig. 4b). These results indicated that, in additional to the HD domain, the putative cleavage site, three other Csm1 motifs, i.e., E415 and C416 C419 in the Linker region and $\mathrm{DxD}$ in the Palm2 domain are also important for the LdCsm DNase. Analysis of the double motif mutants further showed that, while combined mutations of Csm $1^{\text {dHD_LinEC }}, \quad C s m 1^{\text {dHD_LinCC }}$ and Csm1 ${ }^{\mathrm{dHD}}{ }^{\mathrm{P} 2 \mathrm{DD}}$ possessed similar DNase activities relative to those of single motif mutants, those of $\mathrm{Csm}^{\mathrm{dHD}}{ }^{\mathrm{H}}{ }^{\mathrm{P} 2 \mathrm{DxD}}$ and Csm1 $1^{\text {LinE_P2DxD }}$ completely abolished the LdCsm DNase cleavage (Fig. 4b). To this end, we concluded that three Cas10 motifs, including HD, E415, C416 and C419 (Zinc finger) in the Linker as well as D451and D453 (DxD) in Plam2, play critical roles in the LdCsm DNA cleavage.

To yield a further insight into the LdCsm DNA cleavage, all these Csm1-mutated effector complexes were tested for substrate binding in which 25,50 , or $100 \mathrm{nM}$ effector complex was mixed with $500 \mathrm{nM}$ CTR and $5 \mathrm{nM}$ radiolabeled S1-60 ssDNA. After incubation for $3 \mathrm{~min}$, the formation of LdCsm-ssDNA complexes was checked by nondenaturing PAGE. As shown in Supplementary Fig. S6e, the substrate affinity of these effector complexes fell into three different categories. First, WT and LdCsm_Csm1 $1^{\text {P2DD }}$ showed a strong binding ( $100 \%$ and $\sim 80 \%)$, which is consistent with their unimpaired DNase activity; second, $\mathrm{LdCsm}{ }_{-} \mathrm{Csm} 1^{\mathrm{dHD}}$ and LdCsm_Csm1 $1^{\mathrm{P} 2 \mathrm{DxD}}$ retained $\sim 30 \%$ of the binding capacity of the WT LdCsm effector, whereas the last group included those with mutations in Linker, only exhibiting $5-20 \%$ of substrate binding. Taken together, these data indicated the HD motif, the Zinc finger, and the DxD motif function in facilitating substrate binding of the LdCsm effector complex.

\section{LdCsm1 Linker domain and Palm2 DxD motif function in the allosteric control of the LdCsm DNase}

The establishment of ssDNA binding assay with LdCsm prompted us to investigate how target RNAs could regulate the LdCsm DNase. For this purpose, $400 \mathrm{nM}$ S10 RNA (non-homologous RNA) and $500 \mathrm{nM}$ of PTR, NTR or CTR was mixed with $100 \mathrm{nM} \mathrm{LdCsm}$ and $5 \mathrm{nM}$ of labeled S1-60 DNA individually. After incubation at $37^{\circ} \mathrm{C}$ for $3 \mathrm{~min}$, samples were analyzed by non-denaturing PAGE. We found that, while the WT LdCsm effector showed little DNA-binding activity in the presence of nonhomologous RNA or NTR, PTR greatly enhanced the DNA binding and that activity was further facilitated by CTR for ca. fourfold (Fig. 4c, d), indicating that CTR induces allosteric regulation on substrate binding of LdCsm. These results also indicated that the activity of LdCsm DNase involves the protospacer binding-induced allosteric regulation, in which protospacer region-bound facilitates the ssDNA substrate binding but the complexes remain inactive, and CTR-dependent activation of the LdCsm DNase. Therefore, 3' anti-tag of both CTR and NTR regulate LdCsm DNA binding. 
Analyses of all above Csm1-mutated effector complexes revealed that, while five mutated effectors $\left(\mathrm{LdCsm}{ }_{-} \mathrm{Csm} 1^{\mathrm{P} 2 \mathrm{DD}}, \mathrm{LdCsm}{ }_{-} \mathrm{Csm} 1^{\mathrm{dHD}}, \mathrm{LdCsm}_{-} \mathrm{Csm} 1^{\mathrm{LinCC}}\right.$,

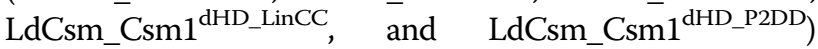
exhibited a pattern of target RNA activation that is very similar to that of the WT LdCsm, those carrying E415A and/or alanine substitutions in Palm2 DxD did not show such a significant CTR-enhanced ssDNA substrate binding; their CTR and PTR ternary complexes basically remained inactive (Fig. 4c, d). These results indicated that, among the three motifs essential for the LdCsm DNase, HD is not involved in CTR-induced allosteric regulation of LdCsm, whereas both E415 and P2-DxD are essential for the allosteric regulation.

Taken together, our results unraveled two distinctive functions for the Cas10 protein: (a) consistent with the results obtained with other type III effectors, the HD motif of LdCsm1 hosts the catalytic site of the DNase; (b) E415 and P2-DxD are involved in mediating allosteric regulation of the ternary effector to yield active enzyme.

\section{Identification of $\mathrm{Csm} 3$ amino acids involved in the regulation of the LdCsm DNase}

The ssDNA substrate-binding assay revealed that the PTR-bound ternary LdCsm showed a higher substrate binding activity compared with LdCsm with nonhomologous RNA (Fig. 4c), indicating that the protospacer region-bound drives certain regulation of LdCsm complex. To yield a further insight into the mechanism, Csm3 and subjected to substitution mutation to yield Csm3 variants, with which mutated effector complexes were purified and characterized in vitro.

Alignment of a selected set of Csm3 proteins revealed a number of conserved amino acids among which many have a structural function or form the intermolecular contacts with crRNA or target RNA (Supplementary Fig. S7). There are three conserved residues: H20, D34, and D106 that do not play a structural role or crRNA or target RNA binding (LdCsm3 number). D34 was the predicted active site of the LdCsm RNase, whereas functions of the remaining two amino acids were elusive. To study that, we constructed several LdCsm3 mutants, including alanine substitution of $\mathrm{H} 20$, D34, or D106 as well as double substitutions (H20/D34 and D34/D106). LdCsm effector complexes carrying each of these Csm3 mutations (designated $\mathrm{Csm}_{3}{ }^{\mathrm{H} 2 \mathrm{OA}}, \mathrm{Csm} 3^{\mathrm{D} 34 \mathrm{~A}}, \mathrm{Csm} 3^{\mathrm{D} 106 \mathrm{~A}}, \mathrm{Csm} 3^{\mathrm{H} 20 /}$ D34-A and $\mathrm{Csm} 3^{\mathrm{D} 34 / \mathrm{D} 106-\mathrm{A}}$ ) were purified from E. coli and characterized. Target RNA cleavage assay showed that, while neither $\mathrm{Csm} 3^{\mathrm{H} 20 \mathrm{~A}}$ nor $\mathrm{Csm} 3^{\mathrm{D} 106 \mathrm{~A}}$ substitutions influenced the target RNA cleavage, Csm $3^{\text {D34A }}$ substitution greatly impaired the RNA cleavage of the effector (Fig. 5a). These results confirmed that D34 is the active site of target RNA cleavage of the LdCsm system as demonstrated for the conserved aspartic acid residue in all other known type III CRISPR-Cas systems. Analysis of RNA-activated ssDNA cleavage showed that the LdCsm complex containing the $\mathrm{Csm} 3^{\mathrm{D} 34 \mathrm{~A}}$ substitution exhibited higher ssDNase activity relative to WT complex, and DNase activity was strongly impaired in the remaining four mutated Csm3 complexes (Fig. 5b).

To investigate their possible roles in spatiotemporal regulation, these Csm3 mutated complexes were incubated with the ssDNA substrate in the presence of different RNAs and analyzed for their substrate binding activity. We found that, while $\mathrm{Csm} 3{ }^{\mathrm{H} 2 \mathrm{OA}}$ substitution impaired ssDNA binding for all tested RNAs, $\mathrm{Csm} 3^{\mathrm{D} 34 \mathrm{~A}}$ exhibited a higher ssDNA substrate-binding enhancement in the presence of PTR vs. non-homologous RNA than WT complex in the presence of either RNA (ca. threefold vs. twofold, respectively), which could be due to the impaired target RNA cleavage that allows a longer persistence of the ternary complex. However, $\mathrm{Csm} 3^{\text {D106A }}$ substitution and the double substitutions $\mathrm{Csm} 3^{\mathrm{H} 20 / \mathrm{D} 34-\mathrm{A}}$ and $\mathrm{Csm} 3^{\mathrm{D} 34 / \mathrm{D} 106-\mathrm{A}}$ did not show such a significant PTRinduced ssDNA substrate-binding enhancement relative to WT complex (Fig. 5c, d). Together, these results confirmed that CTR-activated LdCsm ssDNase activity involves the protospacer binding-induced allosteric regulation, consistent with the results obtained from structural analysis with other Csm or Cmr complexes ${ }^{54,55,59}$. These results also indicated that two $\mathrm{Csm} 3$ residues, $\mathrm{H} 20$ and D106, are involved in mediating regulation of the ternary LdCsm complex to yield active enzyme.

\section{Identification of different target RNA-LdCsm ternary complexes with distinctive substrate binding and DNA cleavage}

To investigate how target RNA could activate the LdCsm DNase, 3'-truncation variants of the CTR were generated, carrying $+1,+2,+3,+4,+5$, and +6 nt of the $3^{\prime}$ anti-tag region of CTR (annotated as $\mathrm{CTR}^{+1}$ to $\mathrm{CTR}^{+6}$ ) as well as the full-length CTR (CTR ${ }^{\text {Full }}$ ) (Supplementary Table S1). Each target RNA was tested for its capability to facilitate DNA binding and DNA cleavage by LdCsm. As shown in Fig. 6a, while the binary LdCsm showed little DNA binding, PTR, the target RNA lacking any $3^{\prime}$ anti-tag sequence, greatly facilitated the substrate binding of the LdCsm complex (ca. 55\% of CTR-LdCsm) but it failed to mediate DNA cleavage. Next, when CTR1, a target RNA that extended the PTR by a nucleotide at the $3^{\prime}$ anti-tag position, was used to form a ternary effector complex with LdCsm, the resulting effector not only showed further increased substrate-binding activity (ca. 90\% of the full activity) but also activated the DNA cleavage (ca. 35\% of full activity). This suggested that a single nucleotide at the $3^{\prime}$ anti-tag of CTR is efficient to convert the inactive ternary LdCsm complex to an active one. Finally, DNA cleavage activity by the LdCsm effector peaked with $\mathrm{CTR}^{+4}$ that 

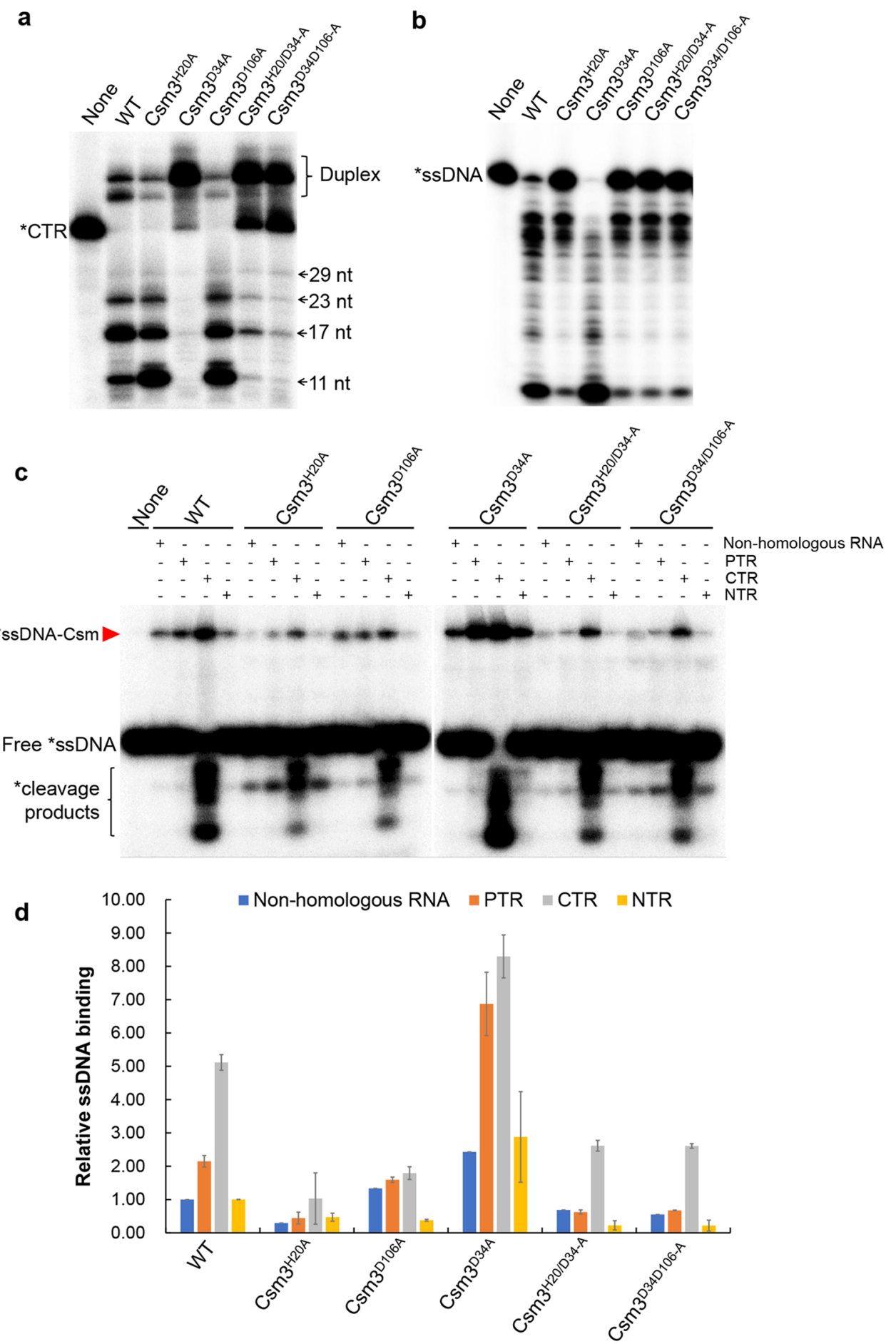

Fig. 5 Effect of LdCsm3 mutations on the ssDNA cleavage and binding of LdCsm. a Target RNA cleavage of LdCsm3 mutated derivatives. Fifty nM of S1-46 RNA were incubated with $50 \mathrm{nM}$ of LdCsm or the indicated mutant derivatives for $10 \mathrm{~min}$, and the samples were analyzed by denaturing PAGE. Duplex: Duplex of crRNA and substrate. b RNA-activated ssDNA cleavage by effectors carrying one of the constructed LdCsm3 mutants. Reaction conditions were the same as in Fig. 4b. c ssDNA binding by effectors carrying each of the constructed LdCsm3 mutants. Reaction conditions were the same as in Fig. 4c. d Relative ssDNA binding between the wild-type LdCsm effector and its LdCsm3 mutated derivatives. The ssDNA activity of LdCsm in non-homologous RNA was used as the standard and set up as 1. Results shown are average of three independent assays; bars represent the mean standard deviation $( \pm S D)$. The red arrow indicates the ssDNA-LdCsm complex. 
a
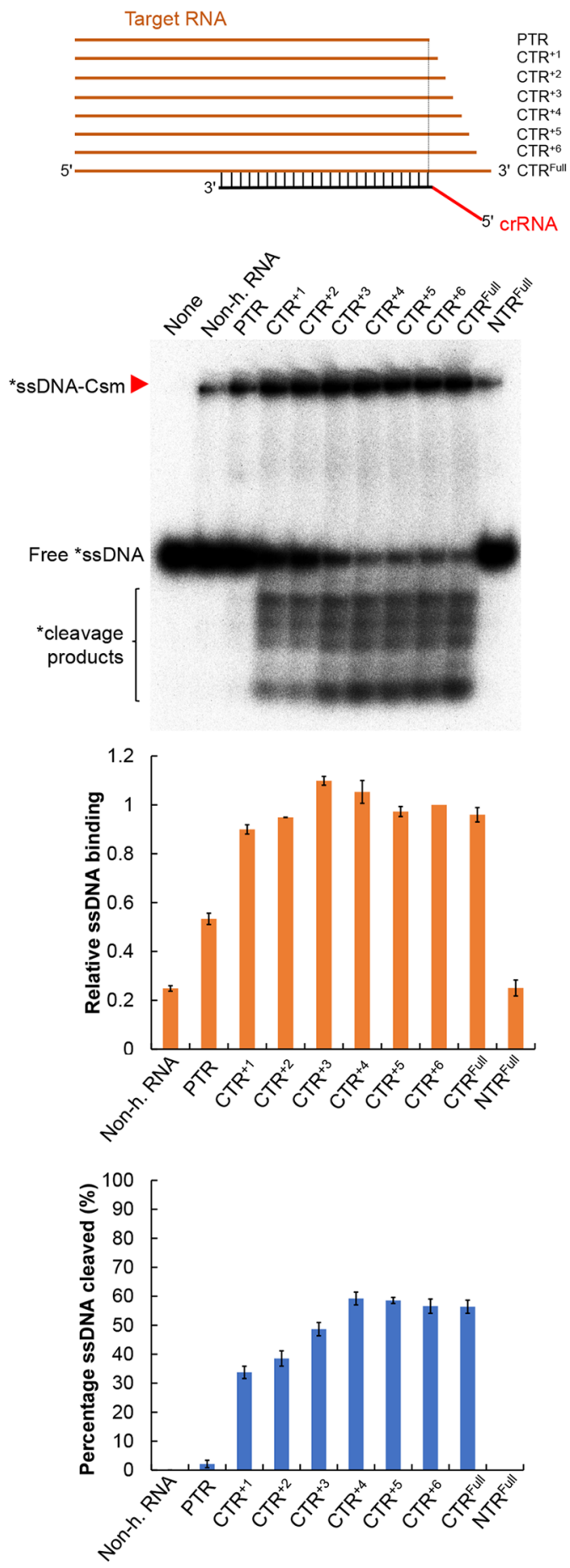

b
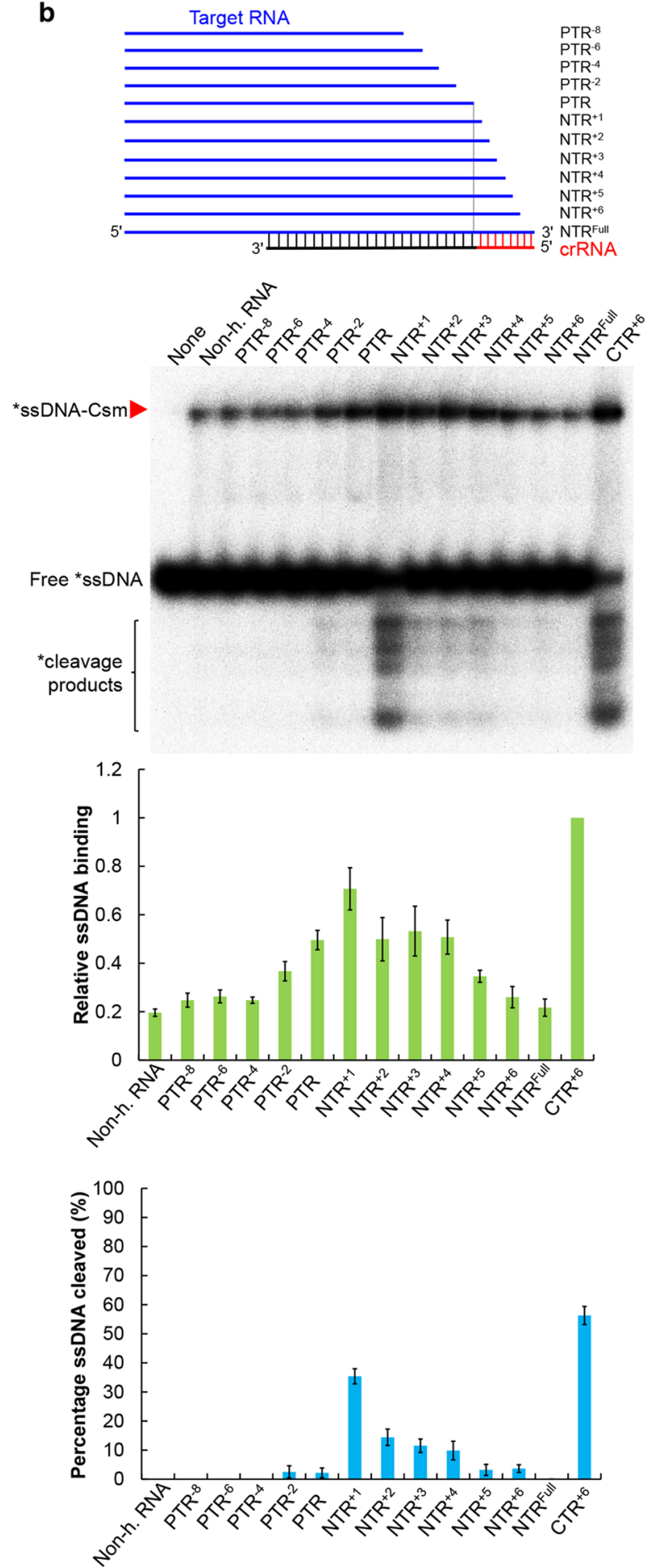

Fig. 6 Target RNA-directed allosteric regulation of LdCsm involves activation and deactivation mechanisms. a CTR activates the LdCsm DNase. b NTR mediates autoimmunity avoidance by deactivation. Reactions were set up with $5 \mathrm{nM} \mathrm{S10-60} \mathrm{ssDNA,} 100 \mathrm{nM}$ of LdCsm, and $400 \mathrm{nM}$ non-homologous RNA (non-h. RNA) or $500 \mathrm{nM}$ target RNA. After addition of one of the target RNAs, the mixture was incubated at $37^{\circ} \mathrm{C}$ for $3 \mathrm{~min}$. Samples were then analyzed by non-denaturing PAGE (in this page) or denaturing PAGE (Supplementary Fig. S8). Red arrowheads indicate the LdCsm-ssDNA complex. Relative ssDNA binding and percentage ssDNA cleaved of LdCsm facilitated by each of these target RNAs were estimated by image quantification of bands on non-denaturing PAGE and denaturing PAGE, using the accessory analysis tool equipped with a Typhoon FLA 7000. For the quantification of the substrate binding, the amount of SSDNA-LdCsm-CTR ${ }^{+6}$ complex is arbitrarily defined as 1 . Results of average of three independent assays are shown with bars representing the standard deviation $( \pm \mathrm{SD})$. 
carries 4 nt 3' anti-tag of CTR, which could have completed the allosteric regulation of the immune system (Fig. 6a).

\section{NTR inhibits the LdCsm DNase by preventing substrate binding}

The same approach was then employed to investigate how NTR could inhibit the LdCsm DNase. Truncated derivatives of NTR were generated (i.e., $\mathrm{NTR}^{+1}-\mathrm{NTR}^{+6}$ ) for the CTR derivatives, and in addition, a few PTR truncations $\left(\mathrm{PTR}^{-8}\right.$ to $\mathrm{PTR}^{-2}$ ) were also made (Supplementary Table S1). These target RNAs were tested for their capability of facilitating substrate binding and cleavage as described above. We found that PTR and its truncated derivatives $\left(\mathrm{PTR}^{-4}-\mathrm{PTR}^{-8}\right)$ showed marginal differences both in ssDNA binding and in ssDNA cleavage, suggesting that these ternary complexes could belong to the same category, equivalent to the stage of the binary LdCsm effector (Fig. 6b). While PTR ${ }^{-2}$ and PTR showed similar effects on ssDNA binding, $\mathrm{NTR}^{+1}$, however, mediated a major stimulation to the LdCsm DNA binding (ca. 70\% of the full activity), and it also facilitated the DNA cleavage (ca. 35\% of full activity), and in fact, the stimulating effect by $\mathrm{NTR}^{+1}$ is comparable to that of $\mathrm{CTR}^{+1}$ (Fig. 6). These results not only implied that the first nucleotide at the $3^{\prime}$ anti-tag of NTR can still allow activation of the HD nuclease domain but also indicated that the process of repression by NTR shares some common activation with the CTR-dependent activation process of LdCsm DNase. Thereafter, extension of the $3^{\prime}$ anti-tag of NTR greatly reduces the DNA binding and gradually decreases DNA cleavage (Fig. 6b), suggesting that the interaction between $3^{\prime}$ anti-tag of NTR and the $5^{\prime}$ tag of crRNA could have deactivated the enzyme by restricting the accessibility of the ssDNA substrate to the active site.

\section{Discussion}

Type III CRISPR-Cas systems characterized thus far show three different interference activities: target RNA cleavage, RNA-activated indiscriminate DNA cleavage, and cOA synthesis among which the latter two activities are responsible for the DNA interference by these systems, whereas target RNA cleavage plays a regulatory role (see reviews ${ }^{46,47,49}$ ). Here we report a type III-A subtype CRISPR-Cas system that shows robust RNA-activated ssDNA cleavage but lacking cOA synthesis. This is consistent with the fact that the genome of the $L$. delbrueckii subsp. bulgaricus genome does not code for any detectable Csm6 homologs. Interestingly, we find that a plasmidborne LdCsm system is sufficient to mediate interference plasmid clearance in E. coli. This suggests that the LdCsm system probably only utilizes the RNA-activated ssDNase to mediate the antiviral immunity in the original host. We have also explored the simplicity of the LdCsm antiviral mechanism for investigating mechanisms of the DNA cleavage by type III CRISPR-Cas systems, and our research has yielded mechanistic insights into the target RNAmediated activation and repression of the LdCsm DNase.

Structures of several type III effector complexes have been solved ${ }^{54-56,59,60}$, and these analyses have revealed that formation of target RNA-Csm ternary effectors involves a minimal conformational change that is very similar between the CTR ternary effectors and the corresponding NTR ternary ones, although only the former has been activated for DNA cleavage ${ }^{54-56}$. As a result, there is no major structural difference at the catalytic site of active vs. inactive Csm DNases, and the only difference is the $3^{\prime}$ anti-tag sequences of the two different types of target RNAs that are individually placed in different channels in their structures ${ }^{54}$. Furthermore, in the structure of the PTR-StCsm complex, major conformational change also occurs although the target RNA lack any 3' anti-tag. Since structure of a DNA substrate-bound Csm effector complex has not been resolved, how these effectors interact with their DNA substrates remains to unknown. For this reason, the only biochemical criterion to distinguish different effector complexes is to analyze their DNA cleavage activity. In this work, we have established a ssDNA-binding assay for LdCsm, and our detailed analysis of substrate binding for this immune system has revealed that different target RNAs are capable of facilitating differential substrate binding and DNA cleavage to the LdCsm effector. Thus it is very interesting to study how allosteric regulation differentially influences the two activities of the LdCsm DNase.

Structural analysis of StCsm by You et al. ${ }^{54}$ has indicated that the Cas10 Linker domain functions in mediating conformational change. Here we find that that both the DxD motif in Palm 2 and the E415 residue in the Linker domain play an essential role in mediating the allosteric regulation of the LdCsm complex in vitro. To date, detailed functions of the Cas10 linker E415 and P2 DxD motifs have not been studied for other type III CRISPR-Cas systems, although all three amino acids are well conserved in Cas10 proteins (Supplementary Fig. S6a). Therefore, it is of a great interest to investigate whether their functions revealed for LdCsm represent a general mechanism for all type III immune systems.

In this paper, we have presented the detailed biochemical analysis of substrate binding by the LdCsm effector complex, and our analysis shows that the Cas10 Zinc finger modulates the enzyme substrate binding since E415A substitution yielded an enzyme with greatly reduced DNA binding and inactive in DNA cleavage (Fig. 4). As E415 is probably part of the Zinc finger motif, this suggests that the Cas10 Zinc finger domain can have dual function in this immune system, namely, both as an executor to the allosteric regulation and as a regulator to substrate binding ${ }^{46,61}$. The other substrate-binding study 
was conducted by single-molecule fluorescence microscopy analysis of Staphylococcus epidermidis Csm by Wang et al. ${ }^{62}$. They find that Cas10 subunit is locked in a static configuration upon NTR binding in which the DNA-binding pocket of the effector appears to be in a closed form, inaccessible to substrate. However, upon CTR binding, Cas10 exhibits a larger conformational space in the active site ${ }^{62}$. Together with our biochemical data, it is thus plausible to predict that there exists a substrate-binding pocket in the DNase of type III effector complexes although this structural domain remains to be illustrated by their structural analysis.

The possibility of characterization of effector complexes for both substrate binding and DNA cleavage allows us to identify several target RNA-LdCsm effector complexes that exhibit differential activities, and we propose that some of these effector complexes probably represent intermediates for structural analysis to study the molecular mechanisms of the CTR activation or NTR repression of the LdCsm system (Fig. 7). These include: (a) The

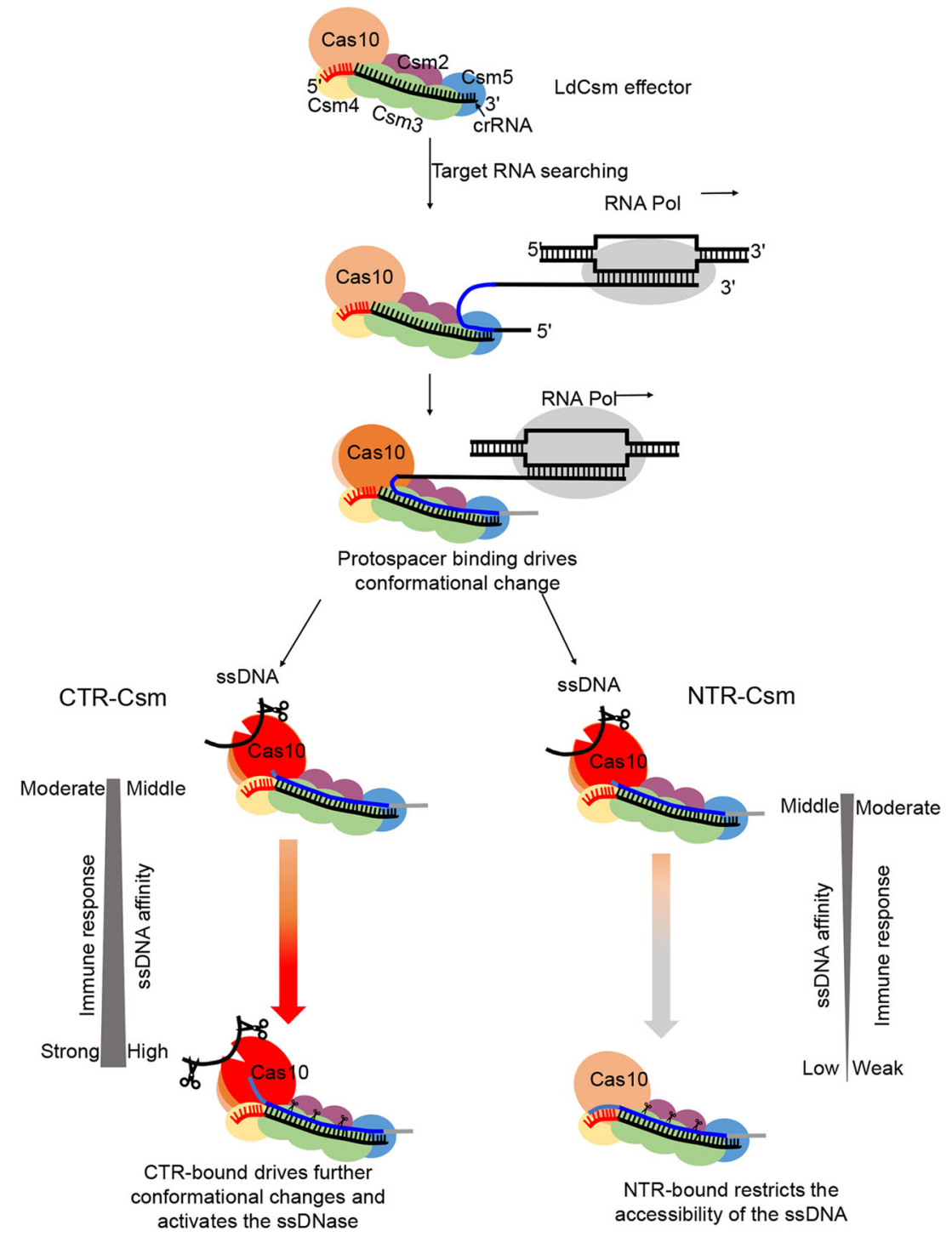

Fig. 7 Model of allosteric activation and repression of the LdCsm DNase. The previous works have proposed the initial recognition of nascent transcript at the 5' end of target RNA for type III complex, since both of Csm5 subunit in Csm complex and Cmr 1 subunit in Cmr complex are crucial for target RNA binding ${ }^{36,37,68}$. These suggested that the binary LdCsm effector complex interacts with target transcript initially at the $5^{\prime}$ end of target RNA and further via sequence complementarity between the protospacer and the corresponding crRNA, leading to the formation of a ternary effector complex with a major conformational change. Addition of a single nucleotide at the 3'-end of protospacer RNA results in an important allosteric change in the LdCsm DNase, giving an active enzyme. CTR-bound LdCsm exhibits the full level of substrate binding and DNA cleavage, whereas NTRbound LdCsm closes the substrate-binding pocket, which deactivates the DNase. Finally, multiple Csm3 subunits cleave the target transcripts, and release of target RNA cleavage products restores the binary conformation, completing the spatiotemporal regulation of LdCsm systems. 
LdCsm-PTR complex showing an elevated level of substrate binding but inactive in catalysis; (b) the LdCsm$\mathrm{CTR}^{+1}$ and LdCsm-NTR ${ }^{+1}$ exhibiting most of substrate binding and catalysis activities, representing another type of intermediates. There are two apparent features with the two effectors: they differ from LdCsm-PTR only by the first nucleotide present in the $3^{\prime}$ anti-tag of target RNAs, and the nucleotide in CTR is different from that in NTR. Thus this nucleotide could functions as a trigger to induce the major allostery in LdCsm ternary to yield an active effector complex and the interaction between the effector with the nucleotide should not be specific; (c) LdCsm$\mathrm{CTR}^{+4}$ represents another effector complex that should have adopted the same conformation since it exhibits the maximal substrate binding and catalysis; and (d) finally LdCsm-NTR ${ }^{\text {Full }}$ represents the inactive conformation of the effector that completely deactivate the LdCsm complex, ensuring autoimmunity avoidance of the LdCsm system (Fig. 7). These LdCsm effector complexes provide good model for structural analysis to reveal the molecular mechanisms of activation and repression of Csm DNases.

\section{Materials and methods}

\section{Bacterial strains and growth conditions}

L. delbrueckii subsp. bulgaricus ND04 (GenBank: CP016393.1) was grown in MRS broth (Oxoid, UK) at $37^{\circ} \mathrm{C}$ without shaking. E. coli JM109 and BL21(DE3) were propagated in Luria-Bertani (LB) medium at $37^{\circ} \mathrm{C}$ with $200 \mathrm{rpm} / \mathrm{min}$ shaking. If applicable, antibiotics were added as the following: ampicillin $(100 \mu \mathrm{g} / \mathrm{ml}$, Sigma), kanamycin $(25 \mu \mathrm{g} / \mathrm{ml}$, Sigma), and chloramphenicol $(10 \mu \mathrm{g} / \mathrm{ml}$, Sigma).

\section{Construction of different vectors and plasmids}

To construct a p15A replicon-based expression vector, the origin fragment was obtained from $\mathrm{pTRKH} 2^{63}$, and ampicillin-resistance gene (Amp) was derived from pUC19 (New England Biolabs), whereas gene expression cassette were amplified from pET30a (Novagen) by PCR, using three sets of primers (p15A-F and p15A-R, Amp-F and Amp-R, as well as LacI-F and T7-R) listed in Supplementary Table S1. Ligation of these DNA fragments generated the expression vector p15AIE (Supplementary Fig. S2a). The same strategy was employed to construct pUCE in which the origin was amplified from pUC19, and chloramphenicol resistance gene was obtained from pCI372, whereas the expression cassette from pET30a, using the primer sets of pUC, $\mathrm{Cm}$, and T7, respectively (Supplementary Table S1). Ligation of these DNA fragments gave pUCE (Supplementary Fig. S2b).

The vector for invader plasmid assay was constructed in two steps. First, fragment 1 containing the origin of pBR322 and kanamycin resistance gene (Kan) was obtained from pET30a by PCR (pBR-Kan primers); second, DNA fragments carrying an arabinose-inducible Bad promoter, multiple cloning sites (MCS), and transcriptional terminator $(\mathrm{T})$ were generated by PCR from $E$. coli BL21(DE3) genome and plasmid $p E L X 1^{64}$, using the primer sets of PBad and MCS-T listed in Supplementary Table S1, respectively, and they were then fused together by splicing overlapping extension PCR (SOE-PCR) ${ }^{65}$; third, ligation of the fragment 1 and the SOE DNA fragment yielded pBad vector. Then three DNA fragments carrying eGFP gene were amplified from pEGFP-N $1^{66}$ by PCR using GFR-F/ GFR-R, CTR-GFR-F/GFR-R, and NTR-GFR-F/GFR-R, and insertion of each fragment into pBad individually gave pBad-G, pBad-CTR, and pPBad-NTR. All primers employed in this work are listed in Supplementary Table S1.

Chromosomal DNA was extracted from cells of $L$. delbrueckii subsp. bulgaricus ND04 using the OMEGA Genomic DNA Purification Kit (OMEGA Bio-tek). DNA fragment covering the cas6-cas10-csm2-csm3-csm4-csm5 gene cassette was amplified by PCR with the primers SalICas6-F and NotI-Csm5-R using ND04 genome DNA as template. The PCR product inserted into p15AIE via SalI and NotI, yielding p15AIE-Cas. csm2 gene was amplified from ND04 genome using primers Csm2-F and Csm2-R. PCR product was then digested with $N d e \mathrm{I}$ and $\mathrm{XhoI}$ and cloned into pET30a expression vectors, giving pET30aCsm2. To construct a CRISPR array plasmid, fusion PCR amplification was performed using three primers Re-S1-F, S1-R1, and Re-S1-R to generate the multiple copies of $36 \mathrm{nt}$ length repeats interspaced by multiple S1 spacer (40 nts) of identical sequence, then PCR products of $\sim 1 \mathrm{~kb}$ were recovered from an agarose gel using an OMEGA GelPurification Kit (OMEGA Bio-tek). The purified DNA fragments were cloned into pJET1.2 (CloneJET PCR Cloning Kit, Thermo Scientific). After confirming the sequence of the synthetic CRISPR array on PJET clones (GATC Biotech), the DNA fragment was amplified and cloned into plasmid pUCE at the BglII site, yielding pUCES1 carried 10 identical spacers (S1) in the CRISPR array. Finally, plasmid p15AIE-Cas-S1 was constructed by insertion of the CRISPR array into p15AIE-Cas at the NheI site.

\section{Purification of LdCsm effector complexes from E. coli}

Three plasmids (p15AIE-Cas, pUCE-S1, and pET30aCsm2) were introduced into $E$. coli BL21(DE3) by electroporation, yielding a $E$. coli strain containing all three plasmids. This bacterial strain was employed as host to overexpress the LdCsm system. The strain was cultured in $200 \mathrm{ml} \mathrm{LB}$ medium containing ampicillin, kanamycin, and chloramphenicol (at $37^{\circ} \mathrm{C}, 200 \mathrm{rpm}$ ) to the mid-log phase $\left(\mathrm{OD}_{600}\right.$ was 0.8 ), then IPTG was added to $0.3 \mathrm{mM}$ and the culture was further cultured at $25^{\circ} \mathrm{C}$ for $16 \mathrm{~h}$. Cells were harvested by centrifugation at $5000 \mathrm{rpm}$ for $5 \mathrm{~min}$, and cell pellets were resuspended in $20 \mathrm{ml}$ buffer A [ $20 \mathrm{mM}$ Tris- $\mathrm{HCl}(\mathrm{pH} 8.5), 0.25 \mathrm{M} \mathrm{NaCl}, 20 \mathrm{mM}$ imidazole and $10 \%$ glycerol], yielding cell suspension that was treated 
with a French press for cell lysis at $4{ }^{\circ} \mathrm{C}$. Cell debris was then removed from treated cell suspension by centrifugation at $10,000 \mathrm{rpm}$ for $30 \mathrm{~min}$ at $4{ }^{\circ} \mathrm{C}$. The $\mathrm{Csm}$ complex was captured on the HiTrap affinity column (GE Healthcare) by LdCsm2 copurification and eluted with buffer B [20 mM Tris- $\mathrm{HCl}(\mathrm{pH}$ 8.5), $0.25 \mathrm{M} \mathrm{NaCl}$, $200 \mathrm{mM}$ imidazole, and $10 \%$ glycerol]. The resulting LdCsm effector complex preparation was further purified by SEC with Superdex 200 (GE Healthcare) using the chromatography buffer $[20 \mathrm{mM}$ Tris- $\mathrm{HCl}$ ( $\mathrm{pH}$ 8.5), $0.25 \mathrm{M} \mathrm{NaCl}$, and $5 \%$ glycerol]. SEC fraction samples were analyzed by SDS-PAGE and those containing the complete set and high quality of Csm complex components were pooled together and used for further analysis. Csm complex concentration was measured according to the Bradford method using the protein assay kit (Thermo Scientific) with bovine serum albumin as the standard.

\section{Extraction and analysis of crRNA}

The purified LdCsm complex $(100 \mu \mathrm{l})$ was first mixed with $100 \mu \mathrm{l}$ Trizol agent (Sigma), and then $200 \mu \mathrm{l}$ chloroform:isoamylalcohol (24:1, v/v) was added. After vortex for $30 \mathrm{~s}$, the mixture was centrifuged at $12,000 \mathrm{rpm}$ for $10 \mathrm{~min}$ at $4{ }^{\circ} \mathrm{C}$. The upper phase was transferred into a new tube and re-extracted with $200 \mu \mathrm{l}$ chloroform:isoamylalcohol. crRNA in the upper phase was precipitated with one volume of isopropanol and washed twice with $1 \mathrm{ml}$ of $70 \%$ ice-cold ethanol. The pellet was air-dried for $30 \mathrm{~min}$ at the room temperature and dissolved in $20 \mu \mathrm{DEPC}-\mathrm{H}_{2} \mathrm{O}$. Ten nanograms of crRNA was 5 -labeled with $\left[\gamma^{-}{ }^{32} \mathrm{P}\right]$-ATP (PerkinElmer) using T4 polynucleotide kinase (New England Biolabs) and separated on a $12 \%$ denaturing polyacrylamide gel. The labeled crRNAs were identified by exposing the gel to a phosphor screen (GE Healthcare) and scanned with a Typhoon FLA 7000 (GE Healthcare). For northern blotting of crRNA, $100 \mathrm{ng}$ of unlabeled crRNA was mixed with equal volume of $2 \times$ RNA loading dye (New England Biolabs) and fractionated in the $12 \%$ denaturing polyacrylamide gel. Northern blotting analysis was conducted as described previously ${ }^{67}$, using radio-labeled RNA S1-40 (Supplementary Table S1).

\section{Labeling of DNA and RNA substrates}

All DNA, S10 (nonhomologous RNA), S1-40 (PTR), S1-46 (CTR), and S1-48 (NTR) oligonucleotides were purchased from IDT, other RNA oligonucleotides were generated by in vitro transcription using the TranscriptAid T7 High Yield Transcription Kit (Thermo Scientific; Supplementary Table S1). DNA and RNA oligonucleotides to be used as substrate for cleavage and binding assays were purified by recovering the corresponding bands from either a native polyacrylamide gel (for double-stranded DNA) or from denaturing polyacrylamide gel (for ssDNA or RNA) after electrophoresis. ssDNA and RNA substrates were $5^{\prime}$ labeled with $\left[\gamma^{-}{ }^{32} \mathrm{P}\right]-\mathrm{ATP}$ and $\mathrm{T} 4$ polynucleotide kinase followed by denaturing gel purification. Double-strand DNA, bubble DNA, and R-loop DNA were generated as described previously ${ }^{31}$.

\section{Cleavage assay}

Nucleic acid cleavage assays were conducted in $10 \mu \mathrm{l}$ of reaction containing the indicated amount of effector complex and substrates in the cleavage buffer $(50 \mathrm{mM}$ Tris-Cl (pH 6.8), $10 \mathrm{mM} \mathrm{MgCl}, 50 \mathrm{mM} \mathrm{KCl}, 0.1 \mathrm{mg} / \mathrm{ml}$ bovine serum albumin (BSA)]. In DNA cleavage assay, $500 \mathrm{nM}$ (unless otherwise indicated) unlabeled RNA was supplemented to activate DNA cleavage activity. Samples were incubated at $37^{\circ} \mathrm{C}$ and stopped for the indicated time periods, and the reaction was stopped by addition of $2 \times$ RNA loading dye (New England Biolabs). For electrophoresis, samples were heated for $3 \mathrm{~min}$ at $95^{\circ} \mathrm{C}$ and analyzed on an $18 \%$ polyacrylamide denaturing gel. RNA ladders were generated by Decade ${ }^{\mathrm{TM}}$ Marker RNA (Ambion) following the instructions and labeled by $\left[\gamma-{ }^{32} \mathrm{P}\right]$-ATP with T4 polynucleotide kinase. Results were recorded by phosphor imaging.

\section{Determination of cyclic oligoadenylate synthesis activity}

Each reaction mixture contained $50 \mathrm{nM}$ Csm complex, $500 \mathrm{nM}$ unlabeled S1-46 RNA (CTR), $\sim 2 \mathrm{nM}\left[\alpha-{ }^{32} \mathrm{P}\right]-$ ATP (PerkinElmer), and an indicated content of ATP in $50 \mathrm{mM}$ Tris-Cl (pH 6.8) and $0.1 \mathrm{mg} / \mathrm{ml}$ BSA supplemented with ions. Reactions were incubated at $37^{\circ} \mathrm{C}$ for $120 \mathrm{~min}$, and $2 \times$ RNA loading dye was added at the indicated time points to stop the reaction. Samples were kept on ice until use. For electrophoresis, samples were treated at $95^{\circ} \mathrm{C}$ for $3 \mathrm{~min}$ and analyzed by $24 \%$ denaturing PAGE. Gels were analyzed by phosphor imaging.

\section{Electrophoretic mobility shift assay}

ssDNA-binding assay was performed by incubating different amounts of Csm complex (specified in each experiment) with $5 \mathrm{nM}{ }^{32} \mathrm{P}-5^{\prime}$-labeled S10-60 ssDNA in the cleavage buffer. All reactions were incubated at $37^{\circ} \mathrm{C}$ for the indicated time periods. Then the same volume of $2 \times$ native loading buffer $[0.1 \%$ bromophenol blue, $15 \%$ sucrose, $\mathrm{w} / \mathrm{v}$ ] was added, and the samples were immediately put on ice and kept there until needed for electrophoresis on an $8 \%$ nondenaturing polyacrylamide gel. Electrophoresis was carried out at $4{ }^{\circ} \mathrm{C}$ using $40 \mathrm{mM}$ Tris and $20 \mathrm{mM}$ acetic acid $\left(\mathrm{pH} 8.4\right.$ at $\left.25^{\circ} \mathrm{C}\right)$ as the running buffer. Gels were analyzed by phosphor imaging.

Relative ssDNA-binding and cleavage activities of LdCsm facilitated by each of these target RNAs were estimated by image quantification of bands on nondenaturing PAGE and denaturing PAGE, respectively, using the accessory analysis tool equipped with a Typhoon FLA 7000. Results of average of three 
independent assays are shown with bars representing the mean standard deviation $( \pm \mathrm{SD})$.

\section{Mutagenesis of LdCsm1 and LdCsm3}

LdCsm 1 mutants were generated using the splicing overlapping extension PCR protocol previously reported ${ }^{63}$. In brief, several mutations were designed in the internal partial overlapping primers, initial PCRs were preformed using the external primer and their corresponding internal primers to generate overlapping gene segments, and then the two PCR products were fused together by overlapping extension PCR. The resulting fragments were digested with restriction endonuclease (DNA fragment containing LdCsm1 H15D16A mutation was cleaved with SalI and SacI, those carrying LdCsm1 E415A, E415C416A, C416C419A, D541D543A, D599D600A, or Q597G were digested with $S t u$ I and SacI). LdCsm3 H20A, D34A, and D106A was obtained via StuI and KpnI. After purification, these DNA fragments were inserted into the plasmid p15AIE-Cas at the corresponding restriction sites, yielding the plasmids carrying each designed $L d C s m 1$ mutation. All mutations were verified by DNA sequencing (GATC Biotech).

\section{Plasmid interference assay}

Plasmid interference assays were performed as previously described ${ }^{51}$. Briefly, an E. coli BL21(DE3) strain carrying p15AIE-Cas-S1 $(80 \mu \mathrm{l})$ was transformed with $100 \mathrm{ng}$ of one of the following plasmids, pBad-G, pBadCTR, or pBad-NTR. Electroporation was performed in a 1 -mm cuvette (Bio-Rad, USA), with the setting of $1600 \mathrm{~V}$, $200 \Omega$, and $25 \mu \mathrm{F}$, using a Gene Pulser II Electroporation System (Bio-Rad). Then $920 \mu \mathrm{l}$ of SOC medium was immediately added to electroporated cells and incubated with shaking $(200 \mathrm{rpm})$ at $37^{\circ} \mathrm{C}$ for $60 \mathrm{~min}$. A series of dilutions were then made for each transformation, and $100 \mu \mathrm{l}$ of each dilution was plated onto LB agar plates containing 0.05 mM IPTG, Ampicillin, Kanamycin, and various concentrations of $\mathrm{L}$-arabinose. Plates were incubated overnight at $37^{\circ} \mathrm{C}$, and the transformation efficiency was calculated. Transformation experiments were conducted for three independent times.

\section{Fluorescence assay}

The reaction mixture $(20 \mu \mathrm{l}$ in total) contains $50 \mathrm{nM}$ complex, the indicated concentration target RNA, in the presence of $500 \mathrm{nM}$ of FAM-poly-16T-BHQ1 ssDNA substrate (Tsingke Biotechnology Company, Wuhan, China). These reactions were incubated in a 384-well black plate (Thermo fisher) and put on a fluorescence plate reader (FLUOstar Omega) for up to $60 \mathrm{~min}$ at $37^{\circ} \mathrm{C}$ with fluorescence measurements taken every 1 min $\left(\lambda_{\text {ex }}\right.$ : $\left.485 \mathrm{~nm} ; \lambda_{\mathrm{em}}: 535 \mathrm{~nm}\right)$. Background fluorescence values were obtained by subtracting fluorescence values obtained from reactions carried out in the absence of target RNA.

\begin{abstract}
Acknowledgements
We thank colleagues at the Archaea Centre for stimulating discussions and Dr. Søren M. Madsen (Bioneer A/S, Hørsholm, Denmark) for a kind gift of pTRKH2 and $\mathrm{pCl} 372$ plasmids. This work has been supported by grants from the National Transgenic Science and Technology Program (2019ZX08010003), Danish Council for Independent Research (DFF-4181-00274), and National Science Foundation of China (31771380) to Q.S.

\section{Author details}

${ }^{1}$ Archaea Centre, Department of Biology, University of Copenhagen, Ole Maaløes Vej 5, 2200 Copenhagen N, Denmark. ${ }^{2}$ College of Life Science and Technology, Huazhong Agricultural University, Wuhan, China. ${ }^{3}$ Key Laboratory of Dairy Biotechnology and Engineering, Inner Mongolia Agricultural University, 010018 Hohhot, China. ${ }^{4}$ Microbial Technology Institute and State Key Laboratory of Microbial Technology, Shandong University, 72 Binhai Road, Jimo, 266237 Qingdao, Shandong, China
\end{abstract}

\section{Author contributions}

H.Z. provided the L. delbrueckii subsp. bulgaricus strain; J.L. and Q.S. designed experiments; J.L. and M.F. performed experiments; J.L., M.F. and Q.S. analyzed data; J.L. and Q.S. wrote the paper. All authors read and approved the final manuscript.

\section{Conflict of interest}

The authors declare that they have no conflict of interest.

\section{Publisher's note}

Springer Nature remains neutral with regard to jurisdictional claims in published maps and institutional affiliations.

Supplementary Information accompanies the paper at (https://doi.org/ 10.1038/s41421-020-0160-4)

Received: 24 December 2019 Accepted: 19 March 2020

Published online: 12 May 2020

\section{References}

1. Barrangou, R. et al. CRISPR provides acquired resistance against viruses in prokaryotes. Science 315, 1709-1712 (2007).

2. Horvath, P. \& Barrangou, R. CRISPR/Cas, the immune system of bacteria and archaea. Science 327, 167-170 (2010).

3. Marraffini, L. A. CRISPR-Cas immunity in prokaryotes. Nature 526, 55-61 (2015).

4. Marraffini, L. A. \& Sontheimer, E. J. CRISPR interference limits horizontal gene transfer in staphylococci by targeting DNA. Science 322, 1843-1845 (2008).

5. van der Oost, J., Westra, E. R., Jackson, R. N. \& Wiedenheft, B. Unravelling the structural and mechanistic basis of CRISPR-Cas systems. Nat. Rev. Microbiol. 12, 479-492 (2014).

6. Wright, A. V., Nunez, J. K. \& Doudna, J. A. Biology and applications of CRISPR systems: harnessing nature's toolbox for genome engineering. Cell 164, 29-44 (2016).

7. Hille, F. et al. The biology of CRISPR-Cas: backward and forward. Cell 172 1239-1259 (2018)

8. Han, W. \& She, Q. CRISPR history: discovery, characterization, and prosperity. Prog. Mol. Biol. Transl. Sci. 152, 1-21 (2017).

9. Koonin, E. V., Makarova, K. S. \& Zhang, F. Diversity, classification and evolution of CRISPR-Cas systems. Curr. Opin. Microbiol. 37, 67-78 (2017).

10. Mohanraju, P. et al. Diverse evolutionary roots and mechanistic variations of the CRISPR-Cas systems. Science 353, aad5147 (2016).

11. Makarova, K. S. et al. An updated evolutionary classification of CRISPR-Cas systems. Nat. Rev. Microbiol. 13, $722-736$ (2015).

12. Marraffini, L. A. \& Sontheimer, E. J. Self versus non-self discrimination during CRISPR RNA-directed immunity. Nature 463, 568-571 (2010).

13. Deng, L., Garrett, R. A., Shah, S. A., Peng, X. \& She, Q. A novel interference mechanism by a type IIIB CRISPR-Cmr module in Sulfolobus. Mol. Microbiol. 87, 1088-1099 (2013) 
14. Hale, C. R. et al. RNA-guided RNA cleavage by a CRISPR RNA-Cas protein complex. Cell 139, 945-956 (2009).

15. Kazlauskiene, M., Tamulaitis, G., Kostiuk, G., Venclovas, C. \& Siksnys, V. Spatiotemporal control of type III-A CRISPR-Cas immunity: coupling DNA degradation with the target RNA recognition. Mol. Cell 62, 295-306 (2016).

16. Peng, W., Feng, M., Feng, X., Liang, Y. X. \& She, Q. An archaeal CRISPR type III-B system exhibiting distinctive RNA targeting features and mediating dual RNA and DNA interference. Nucleic Acids Res. 43, 406-417 (2015).

17. Samai, P. et al. Co-transcriptional DNA and RNA cleavage during type III CRISPR-Cas immunity. Cell 161, 1164-1174 (2015).

18. Tamulaitis, G. et al. Programmable RNA shredding by the type III-A CRISPR-Cas system of Streptococcus thermophilus. Mol. Cell 56, 506-517 (2014).

19. Elmore, J. R. et al. Bipartite recognition of target RNAs activates DNA cleavage by the type III-B CRISPR-Cas system. Genes Dev. 30, 447-459 (2016).

20. Estrella, M. A., Kuo, F. T. \& Bailey, S. RNA-activated DNA cleavage by the type IIIB CRISPR-Cas effector complex. Genes Dev. 30, 460-470 (2016).

21. Manica, A., Zebec, Z., Steinkellner, J. \& Schleper, C. Unexpectedly broad target recognition of the CRISPR-mediated virus defence system in the archaeon Sulfolobus solfataricus. Nucleic Acids Res. 41, 10509-10517 (2013).

22. Zebec, Z., Manica, A., Zhang, J., White, M. F. \& Schleper, C. CRISPR-mediated targeted mRNA degradation in the archaeon Sulfolobus solfataricus. Nucleic Acids Res. 42, 5280-5288 (2014).

23. Goldberg, G. W., Jiang, W., Bikard, D. \& Marraffini, L. A. Conditional tolerance of temperate phages via transcription-dependent CRISPR-Cas targeting. Nature 514, 633-637 (2014).

24. Hale, C. R. et al. capture and cleavage by the Cmr type III-B CRISPR-Cas effector complex. Genes Dev. 28, 2432-2443 (2014).

25. Hale, C. R. et al. Essential features and rational design of CRISPR RNAs that function with the Cas RAMP module complex to cleave RNAs. Mol. Cell 45, 292-302 (2012).

26. Hatoum-Aslan, A., Samai, P., Maniv, I., Jiang, W. \& Marraffini, L. A. A ruler protein in a complex for antiviral defense determines the length of small interfering CRISPR RNAs. J. Biol. Chem. 288, 27888-27897 (2013).

27. Rouillon, C. et al. Structure of the CRISPR interference complex CSM reveals key similarities with cascade. Mol. Cell 52, 124-134 (2013).

28. Staals, R. H. et al. RNA targeting by the type III-A CRISPR-Cas Csm complex of Thermus thermophilus. Mol. Cell 56, 518-530 (2014).

29. Staals, R. H. J. et al. Structure and activity of the RNA-targeting type III-B CRISPRCas complex of Thermus thermophilus. Mol. Cell 52, 135-145 (2013).

30. Zhang, J. et al. Structure and mechanism of the CMR complex for CRISPRmediated antiviral immunity. Mol. Cell 45, 303-313 (2012).

31. Han, W. et al. A type III-B CRISPR-Cas effector complex mediating massive target DNA destruction. Nucleic Acids Res. 45, 1983-1993 (2017).

32. Ichikawa, H. T. et al. Programmable type III-A CRISPR-Cas DNA targeting modules. PLOS One 12, e0176221 (2017).

33. Jiang, W., Samai, P. \& Marraffini, L. A. Degradation of phage transcripts by CRISPR-associated RNases enables type III CRISPR-Cas immunity. Cell 164, 710-721 (2016).

34. Liu, T. Y., lavarone, A. T. \& Doudna, J. A. RNA and DNA targeting by a reconstituted Thermus thermophilus type III-A CRISPR-Cas system. PLOS ONE 12 e0170552 (2017)

35. Park, K. H. et al. RNA activation-independent DNA targeting of the type III CRISPR-Cas system by a Csm complex. EMBO Rep. 18, 826-840 (2017).

36. Li, Y. et al. Cmr1 enables efficient RNA and DNA interference of a III-B CRISPRCas system by binding to target RNA and crRNA. Nucleic Acids Res. 45, 11305-11314 (2017).

37. Pan, S. F. et al. A seed motif for target RNA capture enables efficient immune defence by a type III-B CRISPR-Cas system. RNA Biol. 16, 1166-1178 (2019).

38. Han, W. et al. A type III-B Cmr effector complex catalyzes the synthesis of cyclic oligoadenylate second messengers by cooperative substrate binding. Nucleic Acids Res. 46, 10319-10330 (2018).

39. Kazlauskiene, M., Kostiuk, G., Venclovas, C., Tamulaitis, G. \& Siksnys, V. A cyclic oligonucleotide signaling pathway in type III CRISPR-Cas systems. Science 357, 605-609 (2017)

40. Niewoehner, O. et al. Type III CRISPR-Cas systems produce cyclic oligoadenylate second messengers. Nature 548, 543-548 (2017).

41. Rouillon, C., Athukoralage, J. S., Graham, S., Gruschow, S. \& White, M. F. Control of cyclic oligoadenylate synthesis in a type III CRISPR system. eLife 7, e36734 (2018).
42. Han, W., Pan, S., Lopez-Mendez, B., Montoya, G. \& She, Q. Allosteric regulation of CSx1, a type IIIB-associated CARF domain ribonuclease by RNAs carrying a tetraadenylate tail. Nucleic Acids Res. 45, 10740-10750 (2017).

43. Jia, N., Jones, R., Sukenick, G. \& Patel, D. J. Second messenger cA4 formation within the composite Csm1 Palm pocket of type III-A CRISPR-Cas Csm complex and its release path. Mol. Cell 75, 933-943 (2019).

44. Jia, N., Jones, R., Yang, G., Ouerfelli, O. \& Patel, D. J. CRISPR-Cas III-A Csm6 CARF domain is a ring nuclease triggering stepwise cA4 cleavage with $A p A>p$ formation terminating RNase activity. Mol. Cell 75, 944-956 (2019).

45. Guo, T. et al. Cmr3 regulates the suppression on cyclic oligoadenylate synthesis by tag complementarity in a type III-B CRISPR-Cas system. RNA Biol. 16, 1513-1520 (2019).

46. Tamulaitis, G., Venclovas, C. \& Siksnys, V. Type III CRISPR-Cas immunity: major differences brushed aside. Trends Microbiol. 25, 49-61 (2017).

47. Zhang, Y., Lin, J., Feng, M. \& She, Q. Molecular mechanisms of III-B CRISPR-Cas systems in archaea. Emerg. Top. Life Sci. 2, 483-491 (2018).

48. Varble, A. \& Marraffini, L. A. Three new Cs for CRISPR: collateral, communicate, cooperate. Trends Genet. 35, 446-456 (2019).

49. Pyenson, N. C. \& Marraffini, L. A. Type III CRISPR-Cas systems: when DNA cleavage just isn't enough. Curr. Opin. Microbiol. 37, 150-154 (2017).

50. Molina, R. et al. Structure of $\operatorname{Csx1-COA(4)~complex~reveals~the~basis~of~RNA~}$ decay in type III-B CRISPR-Cas. Nat. Comm. 10, 4302 (2019).

51. Foster, K., Kalter, J., Woodside, W., Terns, R. M. \& Terns, M. P. The ribonuclease activity of Csm6 is required for anti-plasmid immunity by type III-A CRISPR-Cas systems. RNA Biol. 16, 449-460 (2019).

52. Rostol, J. T. \& Marraffini, L. A. Non-specific degradation of transcripts promotes plasmid clearance during type III-A CRISPR-Cas immunity. Nat. Microbiol. 4, 656-662 (2019).

53. Athukoralage, J. S., Rouillon, C., Graham, S., Gruschow, S. \& White, M. F. Ring nucleases deactivate type III CRISPR ribonucleases by degrading cyclic oligoadenylate. Nature 562, 277-280 (2018).

54. You, L. et al. Structure studies of the CRISPR-Csm complex reveal mechanism of co-transcriptional interference. Cell 176, 239-253 (2019).

55. Jia, N. et al. Type III-A CRISPR-Cas Csm complexes: assembly, periodic RNA cleavage, DNase activity regulation, and autoimmunity. Mol. Cell 73, 264-277 (2019).

56. Guo, M. et al. Coupling of ssRNA cleavage with DNase activity in type III-A CRISPR-Csm revealed by cryo-EM and biochemistry. Cell Res. 29, 305-312 (2019).

57. Hatoum-Aslan, A., Maniv, I., Samai, P. \& Marraffini, L. A. Genetic characterization of antiplasmid immunity through a type III-A CRISPR-Cas system. J. Bacteriol. 196, 310-317 (2014).

58. Bhoobalan-Chitty, Y., Johansen, T. B., Di Cianni, N. \& Peng, X. Inhibition of type III CRISPR-Cas immunity by an archaeal virus-encoded anti-CRISPR. Protein Cell 179, 448-458 (2019).

59. Taylor, D. W. et al. Structural biology. Structures of the CRISPR-Cmr complex reveal mode of RNA target positioning. Science 348, 581-585 (2015).

60. Osawa, T., Inanaga, H., Sato, C. \& Numata, T. Crystal structure of the CRISPR-Cas RNA silencing Cmr complex bound to a target analog. Mol. Cell 58, 418-430 (2015).

61. Huo, Y. G. et al. Cryo-EM structure of type III-A CRISPR effector complex. Cell Res. 28, 1195-1197 (2018).

62. Wang, L. et al. Dynamics of Cas10 govern discrimination between self and non-self in type III CRISPR-Cas immunity. Mol. Cell 73, 278-290 (2019).

63. Osullivan, D. J. \& Klaenhammer, T. R. High-copy-number and low-copynumber Lactococcus shuttle cloning vectors with features for clone screening. Gene 137, 227-231 (1993).

64. Chen, Z. et al. Characterization of pMC11, a plasmid with dual origins of replication isolated from Lactobacillus casei MCJ and construction of shuttle vectors with each replicon. Appl. Microbiol. Biotechnol. 98, 5977-5989 (2014).

65. Heckman, K. L. \& Pease, L. R. Gene splicing and mutagenesis by PCR-driven overlap extension. Nat. Protoc. 2, 924-932 (2007).

66. Cormack, B. P., Valdivia, R. H. \& Falkow, S. FACS-optimized mutants of the green fluorescent protein (GFP). Gene 173, 33-38 (1996).

67. Deng, L., Kenchappa, C. S., Peng, X., She, Q. \& Garrett, R. A. Modulation of CRISPR locus transcription by the repeat-binding protein Cbp1 in Sulfolobus. Nucleic Acids Res. 40, 2470-2480 (2012).

68. Mogila, I. et al. Genetic dissection of the type III-A CRISPR-Cas system Csm complex reveals roles of individual subunits. Cell Rep. 26, 2753-2765 (2019). 